1 Universidade Estadual de Campinas (Unicamp) Campinas (SP), Brasil. Orcid: https://orcid. org/0000-0001-98473663

hsatera@gmail.com

2 Rede de Pesquisa em Atenção Primária à Saúde

- Rio de Janeiro (RJ), Brasil. Orcid: https://orcid. org/0000-0002-19821103

inaiara.smsdc@gmail.com

\section{A trajetória da Rede de Pesquisa em Atenção Primária à Saúde da Abrasco}

\author{
The trajectory of the Research Network on Primary Health Care of \\ Abrasco
}

Henrique Sater de Andrade', Inaiara Bragante ${ }^{2}$

DOI: 10.1590/0103-11042018S127

RESUMO A Rede de Pesquisa em Atenção Primária à Saúde (Rede APS) vem buscando, nos últimos oito anos, a participação, o acesso e o intercâmbio de conhecimento científico nacional e internacional sobre Atenção Primária à Saúde (APS) entre pesquisadores, gestores e trabalhadores do Sistema Único de Saúde (SUS). Apresenta-se o contexto de seu surgimento, uma análise cronológica de seus principais marcos históricos e sua relação com o desenvolvimento e com a avaliação de políticas brasileiras de atenção básica. Realizou-se uma análise documental de publicações on-line, documentos oficiais e atas de reuniões e entrevistas com personagens ligados à criação e ao desenvolvimento da Rede. A Rede APS participou diretamente da avaliação de políticas centrais à estruturação da atenção básica brasileira, com destaque para o apoio ao Programa Nacional de Melhoria do Acesso e da Qualidade da Atenção Básica, a difusão de pesquisas sobre o Programa Mais Médicos e dos debates críticos sobre a reformulação da Política Nacional de Atenção Básica. A partir de iniciativas como essa e buscando fortalecer o diálogo e o intercâmbio entre gestores, trabalhadores e pesquisadores do SUS, a Rede pode seguir exercendo um papel relevante na estruturação e na avaliação da APS no País.

PALAVRAS-CHAVE Pesquisa. Atenção Primária à Saúde. Estudos de avaliação.

ABSTRACT Research Network on Primary Health Care (Rede APS) has been seeking for the last 8 years the participation, access and exchange of national and international scientific knowledge on Primary Health Care (APS) among researchers, managers and workers of Unified Health System (SUS). We present the context of its emergence, a chronological analysis of its main historical events and its relationship with the development of policies related to Brazilian APS. We have carried out a documentary analysis of online publications, official documents and meeting reports and interviews with actors related to its creation and development. Rede APS had an important influence on the evaluation of the National Program for Access and Quality Improvement in Primary Health Care (PMAQ-AB), the dissemination of research on the More Doctors Program and in the critical debates about the reformulation of the National Policy of Primary Health Care (PNAB). Based on initiatives such as this one and seeking to strengthen the dialogue and exchange among managers, workers and researchers, this network can continue to play a relevant role in structuring and evaluating APS in Brazil.

KEYWORDS Research. Primary Health Care. Evaluation studies 
A Rede de Pesquisa em Atenção Primária à Saúde (Rede APS) é uma rede vinculada à Associação Brasileira de Saúde Coletiva (Abrasco) que promove, há oito anos, a participação, o acesso e o intercâmbio de conhecimento científico nacional e internacional sobre Atenção Primária à Saúde (APS) entre pesquisadores, gestores e trabalhadores do Sistema Único de Saúde (SUS).

O presente artigo tem como objetivo apresentar o contexto de surgimento da Rede APS, uma análise cronológica de suas principais iniciativas e a relação dela com o desenvolvimento de políticas brasileiras vinculadas à atenção básica. Dentre estas, destacaremos a participação da Rede na formulação e execução do Programa Nacional de Melhoria do Acesso e da Qualidade da Atenção Básica (PMAQ$\mathrm{AB})$, lançado em 2011, na difusão de pesquisas sobre o Programa Mais Médicos para o Brasil (PMM), a partir de 2013, e nos debates críticos sobre a reformulação da Política Nacional de Atenção Básica (PNAB), ocorrida em 2017.

Para isso, realizamos uma análise documental de publicações on-line no site da Rede (rededepesquisaaps.org.br), documentos oficiais e atas das reuniões realizadas pelo seu comitê gestor. Realizamos também entrevistas com personagens diretamente envolvidos em seu lançamento e consolidação nos últimos anos, entre os quais: integrantes do comitê gestor da Rede, o atual coordenador do comitê gestor e a diretora do Departamento de Atenção Básica (DAB) do Ministério da Saúde (MS) no momento de criação da Rede.

\section{Histórico do surgimento da Rede}

O surgimento da Rede APS insere-se em um contexto de avanço da institucionalização da avaliação em saúde, que ocorreu no Brasil, em especial, a partir de 2002. A avaliação de políticas públicas e programas sociais define-se, segundo Contandriopoulos ${ }^{1}$, por:
[...] aplicar um julgamento de valor a uma intervenção, através de um dispositivo capaz de fornecer informações cientificamente válidas e socialmente legítimas sobre ela ou qualquer um dos seus componentes, permitindo aos diferentes atores envolvidos, que podem ter campos de julgamento diferentes, se posicionarem e construírem (individual ou coletivamente) um julgamento capaz de ser traduzido em ação1(706).

No campo da saúde, as pesquisas de avaliação surgem vinculadas aos avanços da epidemiologia e da estatística, testando a utilidade de diversas intervenções e de sistemas de informação que sejam capazes de orientar políticas sanitárias.

Redes de pesquisa no campo da APS foram estabelecidas em países como a Inglaterra para desenvolver e subsidiar a tomada de decisão baseada em evidências científicas. Essas redes podem possibilitar 'coalizões de pesquisadores' para produzir agendas estratégicas de investigação e qualificar a experiência do cuidado na atenção primária².

No Brasil, a avaliação em APS avançou especialmente por meio de iniciativas ao interior do DAB e de projetos como o Projeto de Expansão e Consolidação da Saúde da Família (Proesf), executado pelo DAB a partir de $2003^{3}$. O Proesf tinha como eixo central a expansão da Estratégia Saúde da Família (ESF) e visava qualificar os processos de gestão e trabalho apontando formas de institucionalizar a avaliação em APS; o qual promoveu amplo edital para execução de pesquisas avaliativas nos municípios brasileiros de maior porte ${ }^{4}$.

A partir do avanço da avaliação na atenção básica e de pesquisas avaliativas fomentadas pelo próprio $\mathrm{DAB}$, começaram a surgir propostas de organização de uma rede de pesquisa no âmbito da APS. As primeiras ideias sobre uma rede nesses moldes foram discutidas em 2007, em uma reunião organizada pela Organização Pan-Americana da Saúde (Opas) e pelo MS em Belo Horizonte (MG), 
que incluiu a participação do Ministério da Saúde do Canadá, outras instituições europeias e pesquisadores convidados. É importante ressaltar que o início das discussões ocorreu em momento de avanços na área, um ano após a publicação da primeira PNAB (2006). O Brasil, por contar com uma extensa rede de universidades e instituições de pesquisa com pesquisadores envolvidos em estudos de APS, foi apontado como possível líder no processo de mobilização científica em APS por meio de uma rede de pesquisa ${ }^{5}$.

Com a articulação entre distintas entidades governamentais e acadêmicas, a Rede APS foi lançada três anos mais tarde, em 24 de março de 2010, no Seminário Internacional em APS no Rio de Janeiro. Desde seu lançamento, a Rede é vinculada à Abrasco.

Claunara Mendonça, diretora do DAB na época do lançamento da Rede, afirma que o departamento "tinha interesse em investir em pesquisas sobre APS e já havia feito reuniões com entidades como a Opas nesse sentido". Segundo ela, a ideia inicial era construir uma rede de pesquisas envolvendo todo o continente americano, o que incluiria a apresentação de comunidades de práticas internacionais; mas o projeto não avançou totalmente, e foi mantida e fortalecida a proposta de uma rede brasileira.

Figura 1. Linha do tempo da Rede de Pesquisa em APS

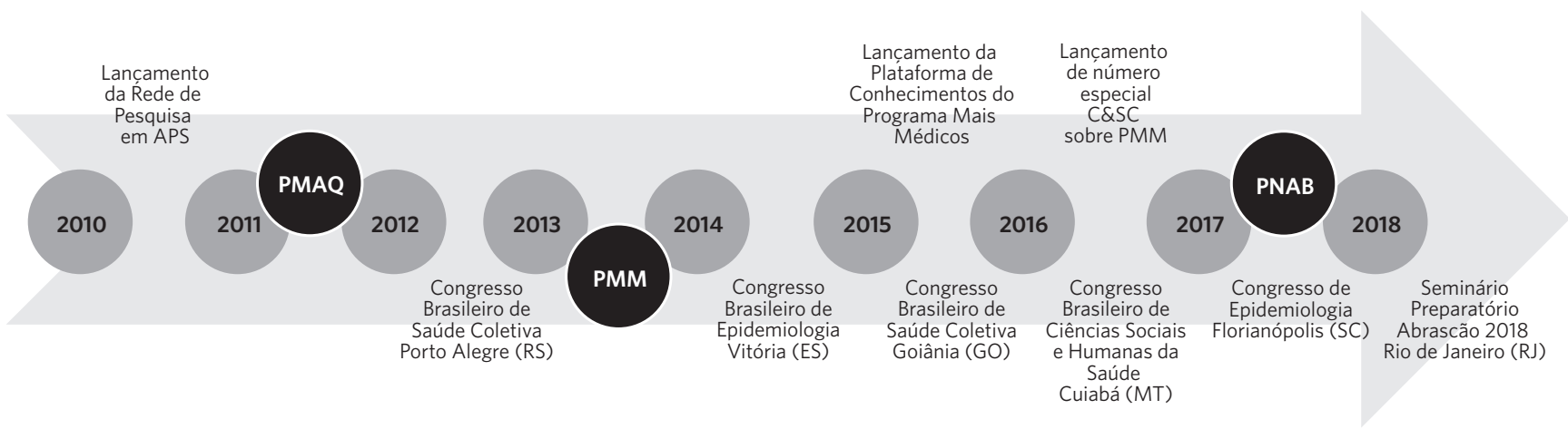

PMAQ: Programa Nacional de Melhoria do Acesso e da Qualidade na Atenção Básica; PMM: Programa Mais Médicos; PNAB: Política Nacional de Atenção Básica.

Luiz Facchini, coordenador da Rede, afirma que a "grande origem da Rede" foi a articulação de um conjunto abrangente de instituições interessadas nas pesquisas de APS e representou uma valorização da pesquisa e da participação das universidades nesse campo. Essa articulação incluiu, além da Abrasco: a Opas; o Conselho Nacional de Secretários de Saúde (Conass); o Conselho Nacional de Secretários Municipais de Saúde (Conasems); a Sociedade Brasileira de Medicina de Família e Comunidade
(SBMFC); a Associação Brasileira de Enfermagem (ABEn) e a Associação Brasileira de Educação Médica (Abem).

Sem um desenho fechado, a Rede busca a participação ampla e a formação de uma comunidade composta por trabalhadores, gestores e pesquisadores vinculados ao SUS. Considera-se que o formato de uma rede contribui para a construção de conexões entre atores envolvidos na avaliação em saúde. Apresenta-se como um modelo promissor para o fortalecimento de pesquisas e 
de formas comuns para a sistematização dos conhecimentos, apesar da ampla variação de atores envolvidos em seus territórios de ação.

De acordo com Rovere ${ }^{\mathbf{6}}$, a consolidação de uma rede depende da fusão de valores como a aceitação e reconhecimento do outro, o interesse nos saberes produzidos, a reciprocidade na colaboração desenvolvida, a solidariedade na cooperação e a confiança na associação. Para que alguém decida entrar para uma rede, é fundamental o exercício cotidiano do encontro e a decisão colaborativa entre os associados.

Nesse sentido, uma rede precisa, para além de uma série de entidades e de indivíduos em contato, da produção de estruturas abertas, capazes de se expandir e integrar novos atores que possam compartilhar os mesmos códigos de comunicação e consigam se comunicar dentro dela ${ }^{7}$.

Desde o início, a Rede APS propôs-se a produzir um espaço apto a construir pontes entre pesquisadores, profissionais e gestores, a ampliar as buscas por informações qualificadas e a aprimorar a avaliação em saúde no âmbito da APS. Além disso, propôs-se a colaborar ativamente para a definição de agendas prioritárias de pesquisas em APS, para a sistematização de informações nacionais sobre o conhecimento da APS, para a tradução de questões de gestores e trabalhadores de APS em pesquisas e de resultados de pesquisas em subsídios para a tomada de decisão informada.

A Rede busca também o intercâmbio de estratégias metodológicas, técnicas e instrumentos de pesquisa entre os atores envolvidos. Para isso, tem como horizonte o fortalecimento do vínculo entre os participantes e o comitê gestor, tanto pelo site e fóruns públicos no ambiente eletrônico como por eventos presenciais.

Seu comitê coordenador conta hoje com 29 pesquisadoras e pesquisadores (17 mulheres e 12 homens), de 11 estados distintos (Acre, Bahia, Ceará, Goiás, Minas Gerais, Paraíba, Rio de Janeiro, Rio Grande do Norte, Rio
Grande do Sul, Santa Catarina, São Paulo) que incluem todas as regiões do País.

Desde seu lançamento, a Rede teve participação ativa nos congressos da Abrasco e buscou construir em suas programações atividades vinculadas a pautas estratégicas relativas à APS, efetuando reuniões, oficinas e seminários nos locais dos eventos. Realizou um total de 25 atividades nacionais coordenadas pelo seu comitê gestor. Nessas oficinas e seminários, foram debatidos, entre outros temas, instrumentos e análises sobre a avaliação em saúde, estratégias metodológicas para a pesquisa em APS, a formação profissional e a gestão e o financiamento da APS, seu papel dentro dos sistemas de saúde, analisados resultados de pesquisas em APS realizadas pelos grupos de pesquisa integrantes da Rede, bem como foram discutidas extensamente variadas políticas e iniciativas vinculadas à atenção básica brasileira.

No período, a Rede, publicou um total de 1.404 postagens em seu site, divulgou mais de 70 editais de pesquisa e realizou 30 entrevistas com personagens relacionados com a APS no País e no mundo. Os boletins são enviados para mais de cinco mil inscritos em sua rede de distribuição, nos quais divulga chamadas para eventos, editais, artigos e outras produções científicas de temas relevantes relacionados à APS.

Nos últimos anos, a Rede apoiou iniciativas de avaliação de políticas governamentais, tendo como um de seus objetivos favorecer o diálogo entre pesquisadores e gestores em APS e informar o debate fundamentado de decisões governamentais no âmbito da APS, proporcionando um espaço de reflexão crítica sobre as políticas de APS no País.

\section{Apoio à avaliação externa do PMAQ-AB}

O PMAQ-AB no Brasil, instituído em 2011, tem como objetivo induzir a ampliação do acesso e da melhoria da qualidade da 
atenção básica, com garantia de um padrão de qualidade comparável nacional, regional e localmente, bem como permitir maior transparência e efetividade das ações na APS ${ }^{8}$.

O Programa inclui em uma de suas fases a avaliação externa, em que são examinados aspectos do acesso e da qualidade por meio de inquéritos com observação in loco e entrevistas com profissionais das equipes de saúde da família sobre a infraestrutura das unidades básicas de saúde, sobre o processo de trabalho das equipes e sobre as experiências de uso e a satisfação dos usuários.

Representantes da Rede APS participaram ativamente do processo de implementação do PMAQ. A avaliação externa desse programa vem sendo realizada por instituições públicas de ensino superior a partir de recomendação da Rede APS para a equipe responsável pelo programa no DAB/MS . Além disso, a Rede mobilizou a expertise de seu comitê gestor para definir perguntas-chave do processo de avaliação. Instituições acadêmicas participantes da Rede, por meio de uma colaboração solidária e intensa, coordenaram a parceria com mais 40 instituições de ensino superior em todo o Brasil, fundamentaram teoricamente o programa e colaboraram para o delineamento dos instrumentos de coleta de dados.

As principais instituições de ensino que participaram do planejamento e que coordenaram o desenvolvimento da avaliação externa no país foram: Universidade Federal de Pelotas (UFPEL); Universidade Federal do Rio Grande do Sul (UFRGS); Universidade Federal do Rio Grande do Norte (UFRN); Universidade Federal da Bahia (UFBA); Universidade Federal de Minas Gerais (UFMG); Escola Nacional de Saúde Pública (Ensp) da Fundação Oswaldo Cruz (Fiocruz).

A visita in loco às equipes de saúde da família e a realização de entrevistas com trabalhadores, gestores e usuários em todas as regiões do Brasil representaram um esforço avaliativo inédito na história da APS no País.
O primeiro ciclo (2011-2012) teve mais de 17 mil equipes certificadas, 65 mil usuários entrevistados e um total de 900 avaliadores externos. Do segundo ciclo (2014-2015), participaram quase 30 mil equipes, 114 mil usuários e 2 mil avaliadores externos.

Parte significativa das reuniões e atividades da Rede APS desse período foi mobilizada no esforço de apoiar a avaliação externa do programa. Em entrevista, Elaine Thumé, professora da UFPEL e integrante do comitê gestor da Rede, detalha esse processo, afirmando que:

Nossa ideia era fazer diferente do que aconteceu no Proesf. Nós buscávamos um instrumento que seria o mesmo para fazer um censo e visitar todas as unidades de saúde da família do Brasil. Nunca tinha havido tamanho esforço de convergência, essas instituições não trabalhavam como parceiras e nesse momento nós buscamos convergir para um esforço nacional de parceria.

Esse instrumento único incorpora-se no processo de institucionalização da avaliação em saúde, tanto no âmbito acadêmico quanto na construção de padrões e indicadores de acesso e de qualidade da gestão e da atenção na APS. Merece destaque o fato de uma rede de pesquisa vinculada especialmente a universidades públicas apoiar um processo de avaliação em saúde. Ao invés de optar pela realização da avaliação externa feita por entidades privadas, o MS, naquele momento, fortaleceu a criação de um corpo de pesquisadores e, sobretudo, uma cultura de pesquisa avaliativa no âmbito da universidade pública e do SUS.

\section{Fortalecimento da avaliação dos efeitos do Programa Mais Médicos a partir de 2013}

O PMM foi instituído em outubro de 2013 com a finalidade de fortalecer a APS no 
Brasil e garantir a provisão de recursos humanos na área médica para o SUS, diminuindo a carência desses profissionais em regiões prioritárias e qualificando a formação médica voltada para a APS no País ${ }^{10}$. O programa previa o provimento emergencial de médicos, a criação de vagas de graduação em medicina, mudanças no âmbito da formação e nas residências médicas e o fortalecimento da atenção básica.

Três anos após seu lançamento, o MS estimava que 18.240 médicos haviam sido contratados pelo programa em 4.058 municípios e 34 distritos indígenas. Essa quantidade de vagas representa cerca de $45 \%$ do total de equipes de ESF no território nacional.

Desde o início, o comitê gestor da Rede APS tomou o PMM e seus efeitos sobre o SUS como um tema prioritário no âmbito da avaliação da APS no País. Em 2015, durante o Congresso Brasileiro de Saúde Coletiva em Goiânia (GO), foi lançada a Plataforma de Conhecimentos do Programa Mais Médicos (http://maismedicos.bvsalud.org/), fruto de uma articulação da Rede com a Opas e com o MS. A plataforma tem como objetivo compartilhar os conhecimentos acumulados no processo de implementação do programa, tornar seus resultados públicos e facilitar o intercâmbio de experiências. Em junho de 2018, o acervo digital da plataforma contava com 428 publicações, sendo 249 artigos, 66 teses e dissertações e 22 monografias.

No início de 2016, o Comitê Gestor da Rede, em parceria com a Opas, divulgou uma chamada pública para publicação de um número temático sobre o PMM na revista 'Ciência \& Saúde Coletiva' da Abrasco. Com o título de 'Pesquisas sobre o Programa Mais Médicos: análises e perspectivas', a edição priorizava estudos relacionados com: a) ampliação de cobertura, acesso e resolubilidade na rede de atenção básica à saúde e em outros níveis do sistema de saúde; b) organização dos sistemas locais de saúde; c) redução de desigualdades e iniquidades em saúde; d) experiências e satisfação de usuários do SUS; e) experiências e satisfação dos médicos do programa; f) experiências institucionais na formação profissional de médicos e mudanças curriculares em cursos de graduação e residência; g) especialização, educação permanente e supervisão presencial de médicos do programa e $h$ ) fontes de informação sobre o PMM e prioridades na avaliação.

Com editoria de integrantes do comitê gestor da Rede, a partir da chamada pública, foram recebidos mais de 70 artigos avaliados por pares. Publicada em setembro do mesmo ano, a edição contou com 23 artigos originais, além de três artigos de opinião e de um debate sobre conquistas e limites do programa ${ }^{12}$.

Além disso, a partir da articulação da Rede APS com a Opas, foram produzidos três relatórios técnicos sobre avaliação e evidências do PMM, relativos a um panorama da produção científican", ao fortalecimento do olhar do gestor e a estratégias metodológicas para a avaliação de impacto do provimento emergencial do PMM.

Com essas iniciativas, a Rede, seguindo seus objetivos de publicizar resultados de pesquisa e promover a gestão de conhecimentos para informar a gestão pública, fortaleceu a avaliação e a reflexão crítica sobre o PMM. Conforme afirmaram os autores do editorial do número temático e membros do comitê gestor da Rede:

[...] consideradas as mudanças e as indefinições recentes do cenário político brasileiro, com iniciativas que atentam contra o direito à saúde e as perspectivas de avanços na construção do SUS, dadas as ameaças de desvinculação de receitas e de constitucionalização de limites para despesas públicas, em contexto de subfinanciamento crônico do SUS, é preocupante a sustentabilidade do PMM. Se quisermos um sistema nacional de saúde com base em uma APS robusta, resolutiva e de qualidade, é essencial garantir a continuidade do PMM, notadamente as estratégias que envolvam o provimento de profissionais e uma formação focada na atuação médica generalista ${ }^{\text {12(2652). }}$. 


\section{Críticas à reformulação da PNAB em 2017}

Editada pela primeira vez em 2006, a PNAB é o principal documento orientador da APS no SUS. Estabelece diretrizes e normas para a organização da atenção básica, para a Estratégia Saúde da Família e para o Programa de Agentes Comunitários de Saúde.

Em 2017, um dos principais debates sobre APS foi a proposta de reformulação da PNAB. Após o anúncio de que a política seria revisada e da divulgação das mudanças propostas, diversas entidades vinculadas ao SUS e ao Movimento da Reforma Sanitária posicionaram-se alertando para os riscos das propostas para uma atenção primária de qualidade no SUS. Em nota publicada pela Abrasco, pelo Centro Brasileiro de Estudos de Saúde (Cebes) e pela Escola Nacional de Saúde Pública Sergio Arouca (Ensp) (Fundação Oswaldo Cruz - Fiocruz), apontavam-se críticas às mudanças no papel dos Agentes Comunitários de Saúde, à implantação de modo reducionista de uma relação de serviços essenciais e estratégicos na APS e nas mudanças nas formas de financiamento da ESF e de equipes tradicionais de atenção básica, com fim da prioridade para o modelo assistencial da ESF13. Após esses questionamentos, em julho de 2017, o MS lançou uma Consulta Pública ${ }^{\mathbf{1 4}}$, abrindo para a população um espaço de avaliações ao texto proposto.

Além dos posicionamentos institucionais, o debate sobre as mudanças na atenção básica do País também chegou à imprensa. Luiz Facchini afirmou à TV Globo, na época, que, com a aprovação das mudanças, recursos que hoje são destinados exclusivamente à ESF serão divididos com outras modalidades de atendimento e fragilizarão essa modalidade bem-sucedida ${ }^{15}$.

Outros membros da Rede foram entrevistados e se posicionaram publicamente contra a reformulação da política. Ligia Giovanella, professora da Ensp/Fiocruz, afirmou ao 'Estadão' que o texto repete várias vezes que a Saúde da Família é estratégica, mas rompe com essa suposta prioridade ao estabelecer que usará verbas específicas para financiar outras formas de atenção básica16(2).

Nesse contexto de ameaças ao SUS, a repercussão do debate evidenciou a importância do tema da APS para o País. Durante o período da consulta pública, a Rede organizou um fórum público para debater a questão. A Consulta Pública chegou a ser prorrogada por mais três dias ${ }^{17}$, devido a mais de cinco mil submissões ao formulário eletrônico.

Mesmo sob protestos, a nova versão da PNAB foi publicada em 21 de setembro de 2017, pela Portaria $\mathrm{n}^{0} 2.436^{18}$. Ainda que o texto final pouco tenha considerado os resultados da consulta pública e os posicionamentos institucionais, avaliou-se que as críticas produzidas no interior da Rede foram importantes para dar visibilidade aos problemas decorrentes da nova política, em especial sobre novas formas de financiamento e na organização assistencial das equipes ${ }^{19}$.

\section{O futuro da Rede APS no contexto de retrocessos para a APS e para o SUS}

\begin{abstract}
A Rede APS vem contribuindo em quase uma década de sua trajetória para o avanço da pesquisa e para a institucionalização da avaliação em saúde no Brasil, em especial no âmbito da APS. Na avaliação de Luiz Facchini:
\end{abstract}

\footnotetext{
A Rede tem capacidades muito promissoras, de interlocução, de multiplicar interesses de outros atores fora da academia. No Brasil, temos um número crescente de trabalhadores e gestores com formação na área de APS e que tem interesse em aumentar sua capacidade de resposta a partir, por exemplo, de estudos de avaliação.
} 
Em março de 2018, a Rede realizou um Seminário Preparatório para o Congresso Brasileiro de Saúde Coletiva Rio de Janeiro, propondo o tema 'De Alma Ata à Estratégia Saúde da Família: 30 anos de APS no SUS - avanços, desafios e ameaças' e com a participação de centenas de pessoas. O seminário recomendou construir uma agenda estratégica a partir de eixos essenciais para a APS no País.

No seminário, foram expostos efeitos positivos $^{\mathbf{2 0}}$ da implementação da APS no Brasil, em especial com a ESF a partir da década de 1990. Foram destacadas, por exemplo, a queda da mortalidade infantil e das internações por condições sensíveis à APS. Foram apresentados também resultados preliminares de um estudo ${ }^{\mathbf{2 1}}$ dos efeitos da recessão econômica e da austeridade fiscal sobre a saúde. Nesse cenário, estima-se cerca de 20 mil mortes infantis e de 124 mil hospitalizações por doenças evitáveis a mais no Brasil até 2030 decorrentes dos cortes nos gastos públicos em APS.

Nesse sentido, o evento trouxe à tona o reconhecimento de que o País vive um momento de graves retrocessos, perda de direitos, ataques à democracia e cortes significativos dos gastos públicos. Apontou o risco de desfiguração da APS, transformada em uma modalidade de serviços básicos de saúde seletivos e de baixíssima qualidade, organizados sob a lógica de atendimento 'queixa-conduta', sem vínculo e responsabilidade sanitária. Destacou-se a necessidade de seguir investigando esse contexto e seus efeitos, a fim de identificar as limitações e contradições da expansão recente da APS no Brasil e a capacidade de sustentação política do avanço de políticas sociais em momentos de crise.

O seminário contou com uma extensa preparação prévia, que incluiu a elaboração e a publicização de textos relacionados com os eixos da agenda e a realização de debates de propostas e estratégias para a APS e para o SUS. As proposições foram reunidas em torno de sete eixos: a) Universalização do acesso à APS resolutiva e de qualidade; b) Agentes Comunitários de Saúde: sujeitos da ação comunitária no território para a promoção da saúde; c) Financiamento suficiente e equitativo para a APS integral; d) Fortalecimento dos espaços democráticos de participação e controle social; e) Mediação de ações intersetoriais para incidir na determinação social, promover a saúde e reduzir as desigualdades; f) Gestão pública democrática, participativa e transparente; g) Formação de profissionais de saúde para a APS integral.

A partir desse processo, foi elaborada uma Agenda Política Estratégica para a APS no SUS, discutida e aprimorada em oficina durante o XII Congresso Brasileiro de Saúde Coletiva. A agenda vem sendo amplamente divulgada e encontra-se publicada neste número temático.

Em síntese, o comitê gestor da Rede APS teve importante influência no desenvolvimento e/ou avaliação de duas das principais políticas da atenção básica brasileira (o PMAQ e o PMM) no período, e, mais recentemente, articulou-se e manifestou-se criticamente ante a revisão da PNAB. A partir de iniciativas como essa e buscando fortalecer o diálogo e o intercâmbio entre gestores, trabalhadores e pesquisadores do SUS, a Rede produziu-se e poderá seguir exercendo um papel relevante na estruturação e na avaliação da APS no País.

\section{Colaboradores}

Andrade HS e Bragante I participaram igualmente da concepção, escrita, formatação e revisão do presente artigo. 


\section{Referências}

1. Contandriopoulos A.P. Avaliando a institucionalização da avaliação. Ciênc Saúde Colet. 2006; 11(3):70511.

2. Thomas P. Networks for research in primary health care. BMJ. 2001; 322(7286):588-90.

3. Almeida PF, Giovanella L. Avaliação em Atenção Básica à Saúde no Brasil: mapeamento e análise das pesquisas realizadas e/ou financiadas pelo Ministério da Saúde entre os anos de 2000 e 2006 . Cadernos de Saúde Pública. 2008; 24(8):11727-1742.

4. Brasil. Ministério da Saúde. Projeto de Expansão e Consolidação da Saúde da Família [internet]. [acesso em 2018 maio 21]. Disponível em: http://189.28.128.100/dab/docs/sistemas/proesf/ apresentacao_PROESF_Geral.pdf.

5. Sampaio LFR. Como surgiu a ideia de criar uma rede de pesquisa em atenção primária à saúde (APS) [internet]. [acesso em 2018 maio 15]. Disponível em: http://rededepesquisaaps.org.br/2010/07/30/dr-luis-fernando-rolim-sampaio/.

6. Rovere M. Redes en Salud: un nuevo paradigma para el abordaje de las organizaciones y la comunidad. Rosario: Ed. Secretaría de Salud Pública, Instituto Lazarte; 1999.

7. Castells M. A sociedade em rede. São Paulo: Paz e Terra; 1999.

8. Brasil Ministério da Saúde. Portaria no ${ }^{\circ}$ 1.654, de 19 de julho de 2011. Institui, no âmbito do Sistema Único de Saúde, o Programa Nacional de Melhoria do Acesso e da Qualidade da Atenção Básica (PMAQ-AB) e o Incentivo Financeiro do PMAQ-AB, denominado Componente de Qualidade do Piso de Atenção Básica Variável - PAB Variável [internet]. Diário Oficial da União. 16 Jul 2011. [acesso em 2018 set 13]. Disponível em: http://189.28.128.100/dab/docs/portaldab/documentos/pmaq/prt_1654_19_07_2011. pdf.
9. Almeida PF, Lima JG. Programa Nacional de Melhoria do Acesso e da Qualidade da Atenção Básica. In: Kalinowski CE, Crozeta K, Costa MFBNA, organizadores. PROENF Programa de Atualização em Enfermagem: Atenção Primária e Saúde da Família: Ciclo 4. Porto Alegre: Artmed Panamericana; 2016. p. 53-98. (Sistema de Educação Continuada a Distância, v. 4).

10. Brasil. Lei $\mathrm{n}^{\mathrm{o}}$ 12.871, de 22 de outubro de 2013 [internet]. Institui o Programa Mais Médicos, altera as Leis no 8.745, de 9 de dezembro de 1993, e no 6.932, de 7 de julho de 1981, e dá outras providências. Diário Oficial da União. 23 Out 2013 [acesso em 2018 maio 5]. Disponível em: http://www.planalto.gov. br/ccivil_03/_ato2011-2014/2013/lei/112871.htm.

11. Organização Pan-Americana da Saúde. Programa Mais Médicos no Brasil: panorama da Produção Científica. Brasília, DF: OPAS; 2017.

12. Facchini LA, Batista SR, Silva Jr AG, et al. O Programa Mais Médicos: análises e perspectivas. Ciênc Saúde Colet. 2016; 21(9):2652-2652.

13. Contra a reformulação da PNAB - nota sobre a revisão da Política Nacional de Atenção Básica [internet]. 2017 [acesso em 2018 maio 9]. Disponível em: https://www.abrasco.org.br/site/outras-noticias/ notas-oficiais-abrasco/contra-reformulacao-da-pnab-nota-sobre-revisao-da-politica-nacional-de-atencao-basica/29798/.

14. Brasil. Ministério da Saúde. Minuta de aperfeiçoamento da PNAB entra em Consulta Pública [internet]. 2017 [acesso em 2018 maio 16]. Disponível em: http://dab.saude.gov.br/portaldab/noticias. php? conteudo=_\&cod $=2426$.

15. TV Globo. Ameaça de corte de verba do Saúde da Família gera reação [internet]. Bom Dia Brasil. 2017 [acesso 2018 maio 6]. Disponível em: https://globoplay.globo.com/v/6055097/programa/. 
16. Lindner J, Castro F. Governo federal quer mudar modelo de atendimento básico de saúde no País [internet]. 2017 [acesso em 2018 maio 10]. Disponível em: https://saude.estadao.com.br/ noticias/geral,governo-federal-quer-mudar-modelo-de-atendimento-basico-de-saude-no-pais,70001932322.

17. Brasil. Ministério da Saúde. Prorrogado prazo da Consulta Pública da PNAB [internet]. 2017 [acesso em 2018 maio 12]. Disponível em: http://dab.saude.gov.br/portaldab/noticias. php? conteudo $=\_\& \operatorname{cod}=2435$.

18. Brasil Ministério da Saúde. Portaria no 2436/GM/ MS, de 21 de setembro de 2017 [internet]. Aprova a Política Nacional de Atenção Básica, estabelecendo a revisão de diretrizes para a organização da Atenção Básica, no âmbito do Sistema Único de Saúde (SUS). Diário Oficial da União. 21 Set 2017. [acesso em 2018 set 17]. Disponível em: http://bvsms.saude. gov.br/bvs/saudelegis/gm/2017/MatrizesConsolidacao/comum/250584.html.
19. Rede de Atenção Primária à Saúde. Sob protestos, texto da nova Política Nacional de Atenção Básica é aprovado [internet]. 2017 [acesso 2018 maio 10]. Disponível em: http://rededepesquisaaps.org. br/2017/09/04/sob-protestos-texto-da-nova-politica-nacional-de-atencao-basica-e-aprovado/.

20. Aquino R. Impactos positivos da atenção primária à saúde na saúde da população [internet]. [acesso em 2018 maio 1]. Disponível em: https://www.abrasco. org.br/site/wp-content/uploads/2018/03/Seminario-APS_Rosana-Aquino.pdf.

21. Rasella D, Basu S, Hone T, et al. Child morbidity and mortality associated with alternative policy responses to the economic crisis in Brazil: A nationwide microsimulation study. PLOS Med. 2018; 15(5):e1002570.

Recebido em 07/06/2018

Aprovado em 21/08/2018

Conflito de interesses: inexistente

Suporte financeiro: não houve 